\title{
Impact of the Concomitant Use of Immunomodulator and a Lower Week 8 Partial Mayo Score on the Persistence of Adalimumab in Refractory Ulcerative Colitis
}

\author{
Shinsuke Kumei ${ }^{1}$, Toshihiro Sakurai ${ }^{2}$, Suketo $\mathrm{So}^{3}$, Soichi Itaba ${ }^{4}$, Hirotada Akiho ${ }^{5}$, \\ Shigeo Nakamura ${ }^{6}$, Hyonji $\mathrm{Kim}^{7}$, Masahiro Yamasaki ${ }^{8}$, Noritaka Takatsu, ${ }^{9,10}$, \\ Ryuichiro Maekawa ${ }^{11}$, Ryosuke Sakemi ${ }^{3}$, Tatsuyuki Watanabe ${ }^{1}$, Michihiko Shibata ${ }^{1}$, \\ Keiichiro Kume ${ }^{1}$, Ichiro Yoshikawa ${ }^{12}$, Yasuhiro Takaki ${ }^{2}$ and Masaru Harada ${ }^{1}$
}

\begin{abstract}
:
Objective Real-world data of adalimumab (ADA) in the treatment of ulcerative colitis (UC) are scarce. We aimed to study the ADA response rates and predictors of response in UC treatment.

Methods This observational, prospective and multi-center study assessed the clinical outcome of refractory UC patients treated with ADA who previously had an inadequate response to either conventional therapies or other anti-TNF antibodies or tacrolimus. The primary endpoint was the proportion of UC patients achieving a clinical response and remission at 8 and 52 weeks. We also evaluated the parameters which were associated with a clinical response at 8 and 52 weeks.

Results A total of 35 patients were enrolled from 11 centers. The clinical responses at 8 and 52 weeks were $60.0 \%$ and $51.4 \%$, respectively. The clinical remission rates at 8 and 52 weeks were $45.7 \%$ and $48.6 \%$, respectively. Positive predictors for week 52 response were combination of ADA with immunomodulator (IM) (OR: 27.229; 95\% CI; 1.897-390.76; p=0.015) and a week 8 lower partial Mayo score (OR: 0.406; 95\% CI; 0.204-0.809; $\mathrm{p}=0.010$ ). A receiver operation characteristic curve analysis revealed the optimal week 8 partial Mayo score to be 2.5, therefore a partial Mayo score of $\leq 2$ was a positive predictor for the continuation of ADA. No malignancy or death occurred during this study.

Conclusion ADA was effective for inducing and maintaining both a clinical response and remission in patients with refractory UC. It remains possible that the concomitant use of IM and a week 8 partial Mayo score of $\leq 2$ may predict the long-term response of ADA.
\end{abstract}

Key words: ulcerative colitis, adalimumab, immunomodulator, partial Mayo score

(Intern Med Advance Publication)

(DOI: 10.2169/internalmedicine.7279-21)

\section{Introduction}

Ulcerative colitis (UC) is a chronic inflammatory disorder of the colon characterized by diarrhea, rectal bleeding, rectal urgency and tenesmus. In Japan, the number of UC patients has been increasing year by year. Estimates based on the issued numbers of certificates of recipients of medical service

\footnotetext{
${ }^{1}$ Third Department of Internal Medicine, University of Occupational and Environmental Health, School of Medicine, Japan, ${ }^{2}$ Department of Gastroenterology, Ashiya Central Hospital, Japan, ${ }^{3}$ Department of Gastroenterology, Tobata Kyoritsu Hospital, Japan, ${ }^{4}$ Department of Gastroenterology, Kyushu Rosai Hospital, Japan, ${ }^{5}$ Department of Gastroenterology, Kitakyushu Municipal Medical Center, Japan, ${ }^{6}$ Department of Gastroenterology, Steel Memorial Yawata Hospital, Japan, ${ }^{7}$ Department of Gastroenterology, JCHO Fukuoka Yutaka Central Hospital, Japan, ${ }^{8}$ Department of Gastroenterology, Kitakyushu General Hospital, Japan, ${ }^{9}$ Department of Gastroenterology, Tagawa Municipal Hospital, Japan, ${ }^{10}$ Department of Gastroenterology, Fukuoka University Chikushi Hospital, Japan, ${ }^{11}$ Department of Gastroenterology, Tagawa Social Insurance Hospital, Japan and ${ }^{12}$ Department of Endoscopy, University of Occupational and Environmental Health, School of Medicine, Japan Received: February 7, 2021; Accepted: April 22, 2021; Advance Publication by J-STAGE: June 12, 2021
} Correspondence to Dr. Shinsuke Kumei, shkumei@med.uoeh-u.ac.jp 
and certificates of registration in 2013 showed that there were over 160,000 patients with UC (approximately 1 case per 1,000 population) (1).

Current treatment options for UC consist of aminosalicylates, corticosteroids, thiopurines, calcineurin inhibitors, anti-tumor necrosis factor (anti-TNF) agents, anti-integrin agents, Janus Kinase inhibitor and interleukin 12/23 inhibitors (2). Although there are lots of agents available for UC treatment, UC remains incurable. It is therefore necessary to use these agents optimally and to maintain high drug persistence.

Adalimumab (ADA) is a recombinant, fully human, monoclonal antibody directed against TNF, and is used as the treatment for moderate-to-severely active UC in adults who had an inadequate response to conventional therapies including corticosteroids and/or thiopurines, or who are intolerant to or have medical contraindications to these therapies $(3,4)$. ADA is approved for self-injection at home in Japan, and it thus reduces the number of hospital visits. ADA is very useful and important especially for young working patients, although some systematic reviews with a meta-analysis have reported that ADA was inferior to other biologics with regard to drug efficacy $(5,6)$.

Drug efficacy, which is assessed by randomized or controlled trials, may substantially differ from its effectiveness in the clinical setting, so real-world studies are necessary to assess the clinical benefit of ADA. We performed a prospective multicenter real-world observational study to evaluate the short-term and long-term effectiveness and safety of ADA, and identified some predictive factors for a higher persistence of ADA.

\section{Materials and Methods}

\section{Study population}

We conducted an observational, prospective and multicenter study including patients from 11 hospitals in the community of Kitakyushu- City in Japan. All refractory UC patients $\geq 13$ years of age treated with ADA following realworld clinical practice considerations (August 2014 to October 2017) were included in this study. The diagnosis of UC was based on the criteria determined by the Japanese Ministry of Health, Labour and Welfare. Consecutive patients with clinically active UC who had an inadequate response to either conventional therapy, including corticosteroids and thiopurines or anti-TNF antibodies or tacrolimus, were assessed for eligibility to be included in this study. There were no criteria about immunomodulator (IM) use, and the attending physician could decide on the use of concurrent IM if necessary. The exclusion criteria were as follows: patients with a contraindication for ADA (severe infection, active mycobacterial infection, known hypersensitivity to excipients of ADA, a past or present history of central nervous system demyelinating disease, or congestive heart failure); patients from whom informed consent could not be obtained; pa- tients with a malignant neoplasm; patients regarded as being inappropriate by the attending physician. The protocol was approved by the institution review board of each center, and each patient provided their written consent.

The information was obtained from personal interviews and each hospital's medical records. Patient's gender, age, weight, disease duration, UC phenotype, hematological parameters, previous and current concomitant medications were registered. The partial Mayo score for UC was used to assess the disease activity.

All patients received $160 \mathrm{mg}$ of ADA subcutaneously at week $0,80 \mathrm{mg}$ at week 2 , and then $40 \mathrm{mg}$ every other week beginning at week 4 .

\section{Outcomes and definitions}

The primary endpoint was the proportion of UC patients achieving a clinical response and remission at 8 and 52 weeks. The clinical response was defined as a composite of : (a) a partial Mayo score reduction of 3 or more accompanied by a decrease in at least $30 \%$ from baseline and (b) a bleeding subscore reduction of 1 or more from baseline, or a bleeding subscore of $\leq 1$. Clinical remission was defined as a partial Mayo score of $\leq 2$ with individual subscore of $\leq 1$ (4). The secondary endpoint was the parameters which were associated with clinical response at 8 and 52 weeks.

In this study, patients who discontinued ADA due to adverse events or stopped attending hospital visits were considered non-responders even if they achieved a clinical response or remission.

\section{Statistical analysis}

Continuous variables with normal distributions were expressed as the mean \pm standard deviation (SD) and compared using Student's $t$-test, and those without normal distributions were expressed as median (interquartile range $(\mathrm{IQR})$ ) and compared using the Mann-Whitney U test between responders and non-responders. Categorical variables were analyzed using the $\chi^{2}$-test or Fisher's exact test between the 2 groups. Univariate and multivariate logistic regression analyses were used to identify the independent predictors of a clinical response at 8 and 52 weeks and were reported as odds ratios (OR) with $95 \%$ confidence intervals (CI). Variables with a $\mathrm{P}<0.10$ according to a univariate analysis were included in the multivariate analysis. The Kaplan-Meier method and log-rank test were used to assess ADA continuation over time. All statistical analyses were conducted using the IBM SPSS version 25.0 software program. P-values less than 0.05 were considered to be statistically significant.

\section{Results}

\section{Baseline patient characteristics}

Between August 2014 and October 2017, 40 patients with refractory UC were treated with ADA at our hospitals. After excluding 5 patients due to missing data, 35 patients were 


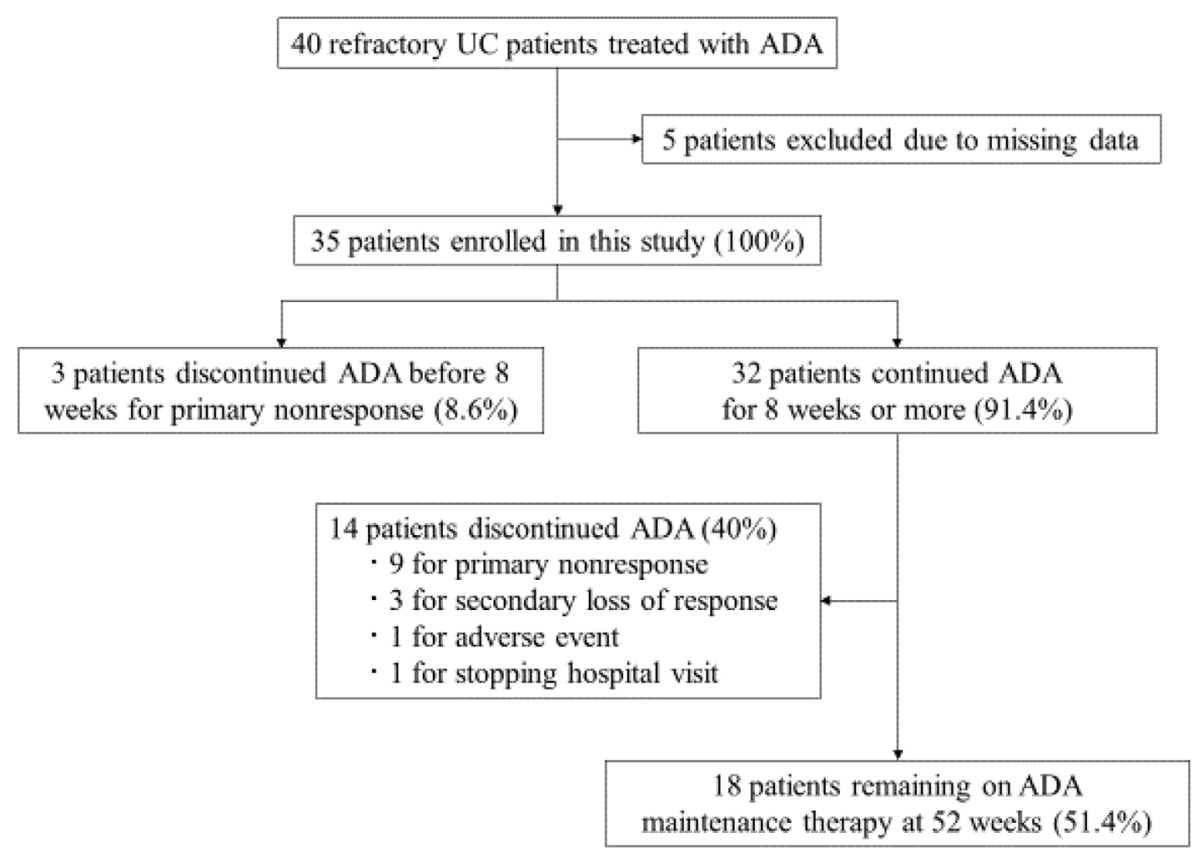

Figure 1. Flow chart of patients inclusion. UC: ulcerative colitis, ADA: adalimumab

Table 1. Baseline Patient Demographics, History and Disease Activity.

\begin{tabular}{lc}
\hline Male, $\mathrm{n}(\%)$ & $19(54.2)$ \\
Age, mean $\pm \mathrm{SD}(\mathrm{years})$ & $41.1 \pm 17.7$ \\
Weight, mean $\pm \mathrm{SD}(\mathrm{kg})$ & $58.6 \pm 12.7$ \\
Duration of UC, median (IQR) (years) & $3.5(0.25-25)$ \\
Extent of Disease, $\mathrm{n}(\%)$ & \\
$\quad$ Left-side & $12(34.3)$ \\
$\quad$ Extensive & $23(65.7)$ \\
Concomitant treatment, $\mathrm{n}(\%)$ & \\
5-aminosalicylic acid & $27(77.1)$ \\
$\quad$ Corticosteroid & $20(57.1)$ \\
$\quad$ Immunomodulator & $13(37.1)$ \\
$\quad$ Cytapheresis & $6(17.1)$ \\
Previous biologic exposure, $\mathrm{n}(\%)$ & \\
$\quad$ Infliximab & $5(14.3)$ \\
Previous calcineurin inhibitor exposure, $\mathrm{n}(\%)$ & \\
$\quad$ Tacrolimus & $5(14.3)$ \\
Disease activity & \\
$\quad$ C-reactive protein, median (IQR) (mg/dL) & $0.66(0.01-11.7)$ \\
$\quad$ Haemoglobin, mean \pm SD (g/dL) & $12.5 \pm 2.2$ \\
$\quad$ Partial Mayo score, median (IQR) & $6(2-9)$ \\
\hline SD: standard deviation, IQR: interquartile range, UC: ulcerative colitis
\end{tabular}

included in this study (Fig. 1). The characteristics of the patients are shown in Table 1. Nineteen patients (54.3\%) were male and the average age was 41.1 years old (SD, 17.7). The median disease duration since diagnosis was 3.5 years (IQR, 0.25-25). Twelve patients (34.3\%) had left-sided colitis and 23 patients $(65.7 \%)$ had extensive colitis. Twenty patients $(57.1 \%)$ had received corticosteroids at the start of ADA, and corticosteroids were tapered after the administration of ADA. Twenty-four patients $(68.6 \%)$ were steroid dependent and 10 patients $(28.6 \%)$ were steroid refractory.
Azathioprine was used as IM in 13 patients (37.1\%): 12 patients had received before administering ADA and 1 patient after administering ADA. The duration of AZA treatment before the administration of ADA in these 12 patients was unknown. Of the 22 patients $(62.9 \%)$ who did not received IM, 1 patient was intolerant to IM. Thirty patients $(85.7 \%)$ were anti-TNF treatment naïve and 5 patients $(14.3 \%)$ had previously received infliximab (IFX), and 3 of 5 patients had also received tacrolimus. The median baseline partial Mayo score was 6 (IQR, 2-9).

\section{Primary endpoint}

The clinical response and remission are shown in Fig. 2. A clinical response was achieved in $60.0 \%$ (21/35) and $51.4 \%(18 / 35)$ patients at 8 and 52 weeks, respectively. Clinical remission was achieved in $45.7 \%$ (16/35) and $48.6 \%$ (17/35) patients at 8 and 52 weeks, respectively. ADA was discontinued in 17 patients (12 for primary nonresponse, 3 for secondary loss of response, 1 for adverse event and 1 for stopping hospital visits) (Fig. 1), and they were considered as non-responders.

\section{Secondary endpoint}

We performed univariate and multivariate logistic regression analyses to identify the independent predictors of a clinical response at 8 and 52 weeks (Table 2). According to a univariate analysis for the clinical response at 8 weeks, there was no significant predictor in baseline variables (data was not shown). A univariate analysis for the clinical response at 52 weeks showed that ADA in combination with IM (OR: 11.786; 95\% CI; 2.041-68.061; $\mathrm{p}=0.006$ ) and the week 8 partial Mayo score (OR: $0.528 ; 95 \%$ CI; 0.332$0.840 ; \mathrm{p}=0.007)$ were significant predictors. A multivariate logistic regression analysis also demonstrated that the sig- 


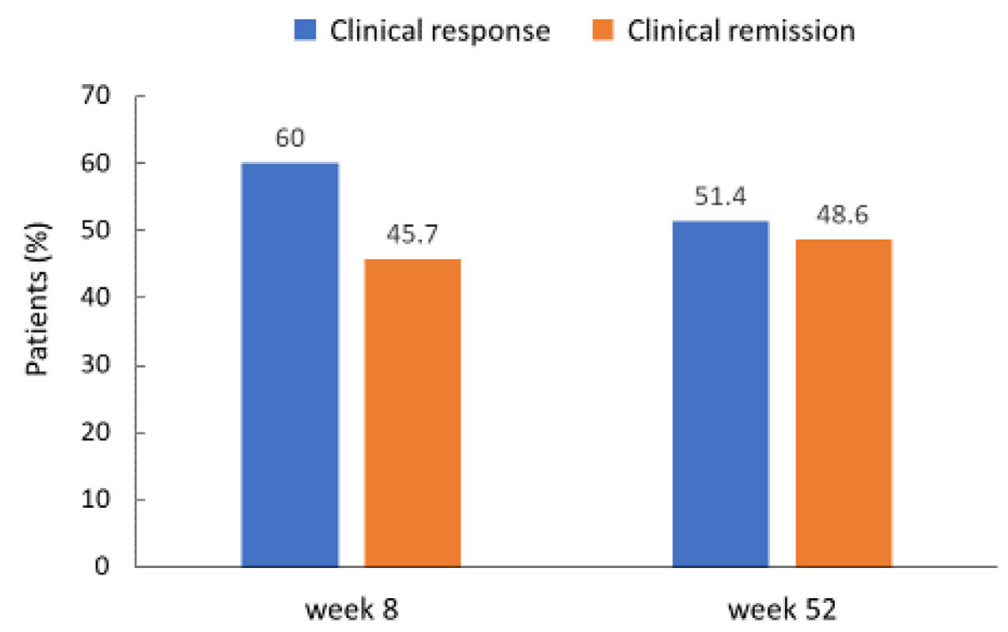

Figure 2. Rates of clinical response and remission at 8 weeks and 52 weeks after starting adalimumab in all patients.

Table 2. Univariate and Multivariate Logistic Regression Analyses to Predict Clinical Response at 52 Weeks.

\begin{tabular}{|c|c|c|c|c|c|c|}
\hline & \multicolumn{3}{|c|}{ Univariate analysis } & \multicolumn{3}{|c|}{ Multivariate analysis } \\
\hline & OR & $95 \% \mathrm{CI}$ & $\mathrm{p}$ value & OR & $95 \% \mathrm{CI}$ & $\mathrm{p}$ value \\
\hline Age & 1.003 & $(0.965-1.041)$ & 0.89 & & & \\
\hline Sex (male/female) & 1.429 & $(0.375-5.437)$ & 0.60 & & & \\
\hline Weight & 1.036 & $(0.975-1.100)$ & 0.28 & & & \\
\hline Duration of UC & 1.004 & $(0.994-1.015)$ & 0.44 & & & \\
\hline Extent of disease (extensive/left-side) & 0.917 & $(0.227-3.704)$ & 0.90 & & & \\
\hline \multicolumn{7}{|l|}{ Concomitant treatment } \\
\hline 5-aminosalicylic acid (yes/no) & 1.077 & $(0.222-5.219)$ & 0.93 & & & \\
\hline Corticosteroid (yes/no) & 0.333 & $(0.082-1.348)$ & 0.12 & & & \\
\hline Immunomodulators (yes/no) & 11.786 & $(2.041-68.061)$ & 0.006 & 27.229 & $(1.897-390.76)$ & 0.015 \\
\hline Cytapheresis (yes/no) & 6.154 & $(0.637-59.466)$ & 0.12 & & & \\
\hline \multicolumn{7}{|l|}{ Previous treatment } \\
\hline Infliximab (yes/no) & 1.500 & $(0.218-10.304)$ & 0.68 & & & \\
\hline Tacrolimus (yes/no) & 4.571 & $(0.456-45.857)$ & 0.20 & & & \\
\hline $\mathrm{CRP}$ at starting $\mathrm{ADA}, \mathrm{mg} / \mathrm{dL}$ & 1.245 & $(0.871-1.780)$ & 0.23 & & & \\
\hline $\mathrm{CRP}$ after 8 weeks, $\mathrm{mg} / \mathrm{dL}$ & 0.900 & $(0.522-1.550)$ & 0.70 & & & \\
\hline Partial Mayo score at starting ADA & 0.905 & $(0.600-1.366)$ & 0.64 & & & \\
\hline Partial Mayo score at 8 weeks & 0.528 & $(0.332-0.840)$ & 0.007 & 0.406 & $(0.204-0.809)$ & 0.010 \\
\hline
\end{tabular}

nificant predictors of a clinical response at 52 weeks were combined treatment with IM (OR: 27.229; 95\% CI; $1.897-$ 390.76; $\mathrm{p}=0.015)$ and the week 8 partial Mayo score (OR: 0.406; 95\% CI; 0.204-0.809; $\mathrm{p}=0.010)$. The baseline clinical characteristics in ADA with IM group and ADA monotherapy group are shown in Table 3 . There was no significant difference between the two groups, except for sex.

Cumulative continuation rates of ADA showed a significant difference between ADA with IM and ADA monotherapy ( $\mathrm{p}=0.005)$ (Fig. 3). A receiver operation characteristic (ROC) curve analysis was performed to determine the optimal cut-off point of week 8 partial Mayo score for clinical response at 52 weeks (Fig. 4). The area under the ROC curve was 0.792 and the cut-off value of the week 8 partial Mayo score was 2.5 with a sensitivity of 0.786 and a speci- ficity of 0.722 . Therefore, a week 8 partial Mayo score of $\leq 2$ was a positive predictor for clinical response at 52 weeks. The cumulative continuation rate of ADA in patients with week 8 partial Mayo score $\leq 2$ showed a significant difference compared with those in patients with week 8 partial Mayo score of $\geq 3$ ( $\mathrm{p}=0.003$ ) (Fig. 5).

\section{Adverse events}

Nine adverse events occurred in 8 out of 35 patients (22.9\%). Most common events were infections (11.4\%). Serious adverse event, associated with the need to interrupt ADA, was experienced by one patient $(2.9 \%)$. This patient developed tuberculosis 8 weeks after ADA induction in spite of negative screening test results, including interferongamma release assay and chest radiograph results prior to 
Table 3. Comparison of the Baseline Characteristics between ADA with IM Group and ADA Monotherapy Group.

\begin{tabular}{|c|c|c|c|}
\hline & $\begin{array}{l}\text { ADA with IM } \\
\quad(n=13)\end{array}$ & $\begin{array}{l}\text { ADA monotherapy } \\
\qquad(\mathrm{n}=22)\end{array}$ & $\mathrm{p}$ value \\
\hline Male, n (\%) & $4(30.8 \%)$ & $15(68.2 \%)$ & 0.03 \\
\hline Age, mean $\pm \mathrm{SD}$ (years) & $47.5 \pm 18.6$ & $37.3 \pm 16.4$ & 0.10 \\
\hline Weight, mean \pm SD $(\mathrm{kg})$ & $54.0 \pm 11.0$ & $60.9 \pm 13.1$ & 0.15 \\
\hline Duration of UC, median (IQR) (years) & $3.5(1.1-25)$ & $3.3(0.25-17.4)$ & 0.93 \\
\hline Extent of Disease, n (\%) & & & 0.29 \\
\hline Left-side & $6(46.2 \%)$ & $6(27.3 \%)$ & \\
\hline Extensive & $7(53.8 \%)$ & $16(72.7 \%)$ & \\
\hline \multicolumn{4}{|l|}{ Concomitant treatment, $\mathrm{n}(\%)$} \\
\hline 5-aminosalicylic acid & $11(84.6 \%)$ & $16(72.7 \%)$ & 0.68 \\
\hline Corticosteroid & $5(38.5 \%)$ & $15(68.2 \%)$ & 0.09 \\
\hline Cytapheresis & $3(23.1 \%)$ & $3(13.6 \%)$ & 0.65 \\
\hline \multicolumn{4}{|l|}{ Previous biologic exposure, $\mathrm{n}(\%)$} \\
\hline Infliximab & $3(23.1 \%)$ & $2(9.1 \%)$ & 0.34 \\
\hline \multicolumn{4}{|l|}{ Previous calcineurin inhibitor exposure, $\mathrm{n}(\%)$} \\
\hline Tacrolimus & $4(30.8 \%)$ & $1(4.5 \%)$ & 0.05 \\
\hline \multicolumn{4}{|l|}{ Disease activity } \\
\hline C-reactive protein, median (IQR) (mg/dL) & $0.36(0.01-11.7)$ & $1.48(0.02-4.9)$ & 0.24 \\
\hline Haemoglobin, mean \pm SD $(g / d L)$ & $11.9 \pm 2.3$ & $12.8 \pm 2.0$ & 0.24 \\
\hline Partial Mayo score, median (IQR) & $5(3-9)$ & $6(2-8)$ & 0.56 \\
\hline
\end{tabular}

ADA: adalimumab, IM: immunomodulator, SD: standard deviation, IQR: interquartile range, UC: ulcerative colitis

ADA induction. No malignancy or death occurred during this study.

\section{Discussion}

This observational, prospective and multi-center study of ADA for refractory UC patients showed that a combination of ADA and IM, and a week 8 partial Mayo score of $\leq 2$ were predictive for a long-term good response.

Several studies have evaluated the effectiveness of combining IFX with IM in patients with moderate-to-severe UC $(7,8)$. However, the effectiveness of combining ADA with IM is unclear (9-13). A retrospective analysis of large cohort by Chen et al. demonstrated that combination therapy using IM can increase the persistence of biologic treatments including ADA (14). To the best of our knowledge, this is the first prospective and multi-center study to suggest that concomitant use of IM with ADA in refractory UC patients may be effective. In Crohn's disease (CD) patients, a multicenter, randomized, prospective, open-label study (DIAMOND trial) by Matsumoto et al. showed that the simultaneous use of IM with ADA may improve the mucosal healing associated with improved long-term outcomes of patients of inflammatory bowel disease (15). A subanalysis of DIAMOND trial by Nakase et al. showed that higher 6thioguanine nucleotide (6-TGN) induced by the optimized use of IM could inhibit the occurrence of anti-adalimumab antibodies (AAA) (16). The appearance of AAA is associated with low trough serum ADA concentrations and a decreased clinical response $(17,18)$. Our study suggested that
IM could decrease AAA and could add anti-inflammatory effects, and together this contributed to the higher persistence of ADA. Similar results have already been reported in the field of rheumatoid arthritis (19).

The decision to continue long-term therapy in patients with UC is generally based on the response to induction therapy (20). In Japan, a multicenter, phase II/III, randomized, double-blind, placebo-controlled study by Suzuki et al. showed the week 8 response to ADA to be associated with greater rates of response, remission, and mucosal healing compared with the overall adalimumab population at week 52 (21). Likewise, our study showed that the week 8 response to ADA was associated with a greater clinical response at 52 weeks. The week 8 lower partial Mayo score was a significant predictor of a clinical response at 52 weeks. Moreover, a week 8 partial Mayo score of $\leq 2$ was found to be a positive predictor to continue ADA.

According to the results of this study, we suggest two treatment strategies when starting ADA in refractory UC patients. First, we should consider the combined use with IM. In the case of patients who have already been administered IM before starting ADA, IM should be continued without interruption. In the case of patients who have not been administered IM, then combination treatment of ADA with IM should be considered, especially when there is loss of response to ADA. Second, we should focus on achieving a week 8 partial Mayo score of $\leq 2$, because our study showed that it was a predictive factor for the long-term response. A partial Mayo score of $\leq 2$ means clinical remission. Therefore, we should aim at achieving clinical remission within 8 


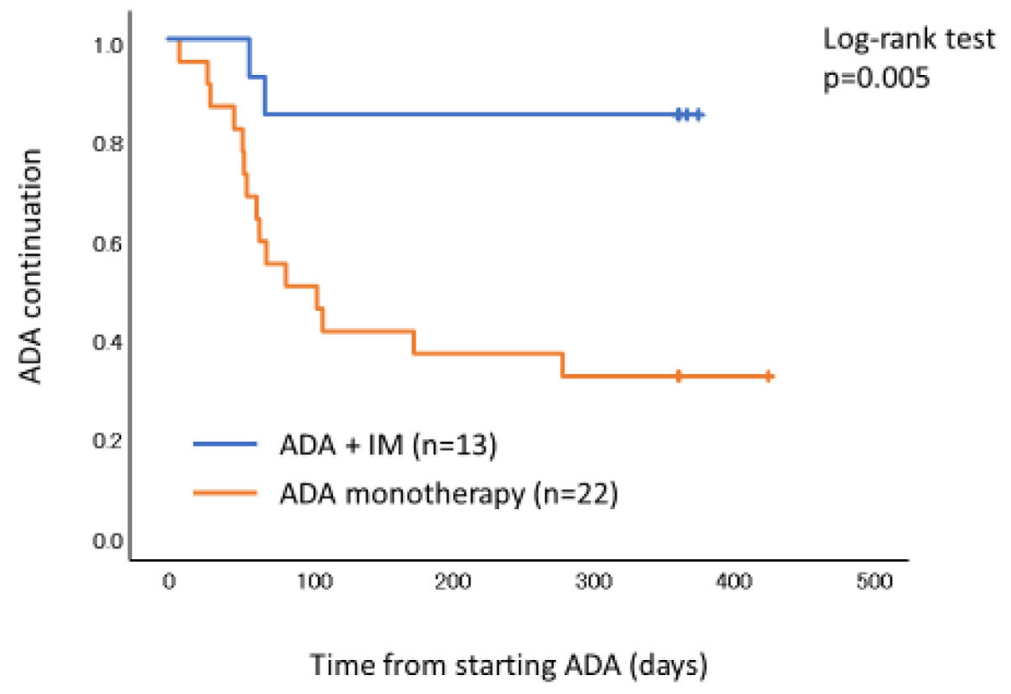

Figure 3. The Kaplan-Meier survival curves of ADA continuation. Patients with ADA and IM were significantly more likely to keep higher persistence of ADA compared to those with ADA monotherapy. ADA: adalimumab, IM: immunomodulator

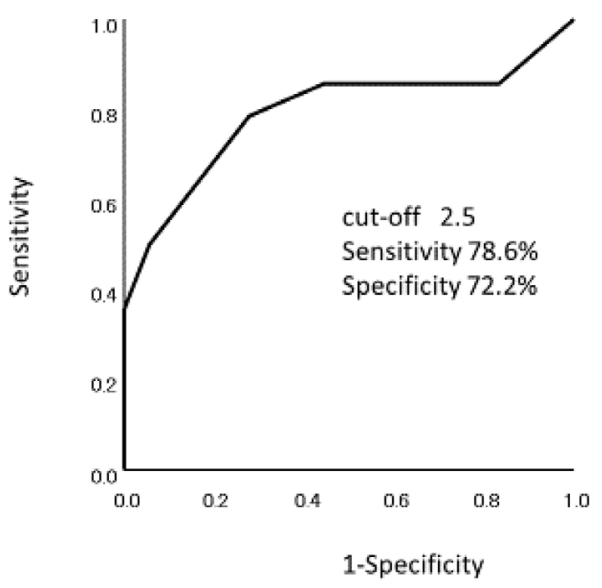

Figure 4. ROC curve to identify the optimal cut-off point of week 8 partial Mayo score for clinical response at 52 weeks. Area under the ROC curve was 0.792 and the cut-off point of week 8 partial Mayo score was 2.5 with a sensitivity 0.786 and a specificity of 0.722 . ROC: receiver operation characteristics

weeks of starting ADA with the optimized use of conventional therapy. This leads to a higher persistence of ADA. In the case of patients who have not achieved clinical remission at 8 weeks, we should therefore consider step-up treatment, including the addition of IM or cytapheresis. If we can easily measure the trough serum ADA concentrations and AAA, it will be easy to decide whether or not we should add IM or switch to other biologics. However, in the real-world, it is difficult to monitor the trough serum ADA concentrations and AAA. On the other hand, the partial Mayo score is a very simple scale and it is used widely as the outcome measure for clinical trials assessing therapy for UC (22). It allows us to assess the patient's condition without endoscopic findings at any time. For this reason, considering the combination use of IM, and referring to the week
8 partial Mayo score, it therefore seems to be important in order to use ADA effectively in daily clinical practice.

This study is associated with several limitations. First, the number of patients was small, and this study may therefore not provide sufficient evidence. Prospective studies with a larger number of patients are needed to confirm these findings. Second, there were few severe UC patients. This might have led to a better response and remission rate in our study than previous randomized placebo-controlled reports like the ULTRA trial $(3,4,20,21)$. In the real-world, it remains possible that ADA is not likely to be used for severe UC patients because some systematic reviews with meta-analyses have reported that ADA was inferior to other biologics for moderate to severe UC patients $(5,6)$. The response and remission rate in our study may thus reflect the real-world situation in which physicians use the appropriate biologics according to each patient's state. Third, endoscopic evaluation was not performed in all patients, and we could not assess the full Mayo score. Fourth, there was no criteria about how to use IM, such as the starting date, duration of administration or dose of IM. Refractory UC patients could participate in this study, regardless of whether they received IM. In this study, IM was used in 13 patients (37.1\%): 12 patients had received IM before administering ADA and 1 patient after administering ADA. AZA treatment before starting ADA may be more effective for UC treatment by ADA. Fifth, there was no data about the trough value of serum ADA concentrations and AAA. However, this also reflects the real-world situation and enabled studying familiar predictive factors such as IM and the partial Mayo score.

In conclusion, we performed an observational, prospective and multi-center study of ADA for refractory UC patients who previously had an inadequate response to either conventional therapy including corticosteroids and thiopurines or other anti-TNF antibodies or tacrolimus. ADA was thus 


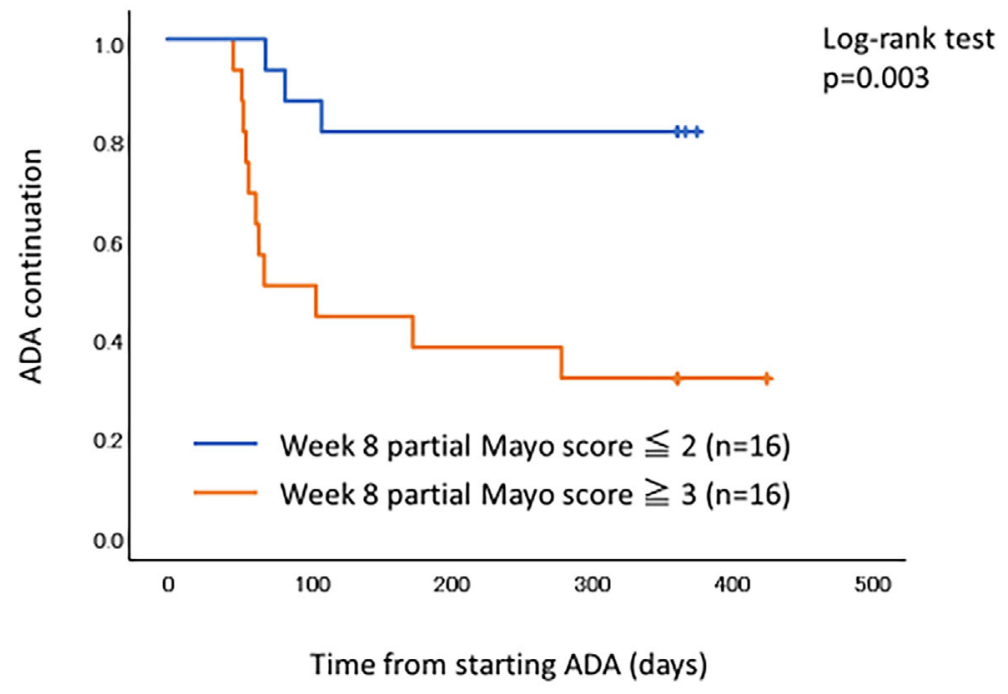

Figure 5. The Kaplan-Meier survival curves of ADA continuation. Patients with week 8 partial Mayo score $\leq 2$ were significantly more likely to keep higher persistence of ADA compared to those with week 8 partial Mayo score $\geq 3$. ADA: adalimumab

found to be effective for inducing and maintaining both a clinical response and remission in patients with refractory UC. Our study suggests that combination treatment with IM and a week 8 partial Mayo score of $\leq 2$ may be predictive for the long-term response of ADA in the real-world clinical setting.

The authors state that they have no Conflict of Interest (COI).

\section{References}

1. Higashiyama M, Sugita A, Koganei K, et al. Management of elderly ulcerative colitis in Japan. J Gastroenterol 54: 571-586, 2019.

2. Feuerstein JD, Isaacs KL, Schneider Y, et al. AGA Clinical Practice Guidelines on the Management of Moderate to Severe Ulcerative Colitis. Gastroenterology 158: 1450-1461, 2020.

3. Reinisch W, Sandborn WJ, Hommes DW, et al. Adalimumab for induction of clinical remission in moderately to severely active ulcerative colitis: results of a randomized controlled trial. Gut $\mathbf{6 0}$ : 780-787, 2011.

4. Sandborn WJ, van Assche G, Reinisch W, et al. Adalimumab induces and maintains clinical remission in patients with moderateto-severe ulcerative colitis. Gastroenterology 142: 257-265, 2012.

5. Vickers AD, Ainsworth C, Mody R, et al. Systematic Review with Network Meta-Analysis: Comparative Efficacy of Biologics in the Treatment of Moderately to Severely Active Ulcerative Colitis. PLoS One 11: e0165435, 2016.

6. Cholapranee A, Hazlewood GS, Kaplan GG, et al. Systematic review with meta-analysis: comparative efficacy of biologics for induction and maintenance of mucosal healing in Crohn's disease and ulcerative colitis controlled trials. Aliment Pharmacol Ther 45: 1291-1302, 2017.

7. Panaccione R, Ghosh S, Middleton S, et al. Combination therapy with infliximab and azathioprine is superior to monotherapy with either agent in ulcerative colitis. Gastroenterology 146: 392-400, 2014.

8. Kobayashi T, Udagawa E, Uda A, et al. Impact of immunomodulator use on treatment persistence in patients with ulcerative colitis. J Gastroenterol Hepatol 35: 225-232, 2020.
9. Bots S, Gecse K, Barclay M, et al. Combination Immunosuppression in IBD. Inflamm Bowel Dis 24: 539-545, 2018.

10. Sultan KS, Berkowitz JC, Khan S. Combination therapy for inflammatory bowel disease. World J Gastrointest Pharmacol Ther 8: 103-113, 2017.

11. Targownik LE, Benchimol EI, Bernstein CN, et al. Upfront combination therapy, compared with monotherapy, for patients not previously treated with biologic agent associates with reduced risk of inflammatory bowel disease-related complications in a populationbased cohort study. Clin. Gastroenterol. Hepatol 17: 1788-1798, 2019.

12. Strik AS, van den Brink GR, Ponsioen C, et al. Suppression of anti-drug antibodies to infliximab or adalimumab with the addition of an immunomodulator in patients with inflammatory bowel disease. Aliment Pharmacol Ther 45: 1128-1134, 2017.

13. Viazis N, Giakoumis M, Bamias G, et al. Predictors of tissue healing in ulcerative colitis patients treated with anti-TNF. Dig Liver Dis 49: 29-33, 2017.

14. Chen C, Hartzema AG, Xiao H, et al. Real-world Pattern of Biologic Use in Patients with Inflammatory Bowel Disease: Treatment Persistence, Switching, and Importance of Concurrent Immunosuppressive Therapy. Inflamm Bowel Dis 25: 1417-1427, 2019.

15. Matsumoto T, Motoya S, Watanabe K, et al. Adalimumab Monotherapy and a Combination with Azathioprine for Crohn's Disease: A Prospective, Randomized Trial. J Crohns Colitis 10: 1259-1266, 2016.

16. Nakase H, Motoya S, Watanabe K, et al. Significance of measurement of serum trough level and anti-drug antibody of adalimumab as personalized pharmacokinetics in patients with Crohn's disease: a subanalysis of the DIAMOND trial. Aliment Pharmacol Ther 46: 873-882, 2017.

17. Karmiris K, Paintaud G, Noman M, et al. Influence of trough serum levels and immunogenicity on long-term outcome of adalimumab therapy in Crohn's disease. Gastroenterology 137: 1628-1640, 2009.

18. Imaeda H, Takahashi K, Fujimoto T, et al. Clinical utility of newly developed immunoassays for serum concentrations of adalimumab and anti-adalimumab antibodies in patients with Crohn's disease. J Gastroenterol 49: 100-109, 2014.

19. Soliman MM, Ashcroft DM, Watson KD, et al. Impact of concomitant use of DMARDs on the persistence with anti-TNF thera- 
pies in patients with rheumatoid arthritis: results from the British Society for Rheumatology Biologics Register. Ann Rheum Dis 70: 583-589, 2011.

20. Sandborn WJ, Colombel J-F, D'Hanes G, et al. One-year maintenance outcomes among patients with moderately-to-severely active ulcerative colitis who responded to induction therapy with adalimumab: subgroup analyses from ULTRA 2. Aliment Pharmacol Ther 37: 204-213, 2013.

21. Suzuki $Y$, Motoya $S$, Hanai $H$, et al. Efficacy and safety of adalimumab in Japanese patients with moderately to severely active ulcerative colitis. J Gastroenterol 49: 283-294, 2014.

22. Lewis JD, Chuai S, Nessel L, et al. Use of the Non-invasive Components of the Mayo Score to Assess Clinical Response in Ulcerative Colitis. Inflamm Bowel Dis 14: 1660-1666, 2008.

The Internal Medicine is an Open Access journal distributed under the Creative Commons Attribution-NonCommercial-NoDerivatives 4.0 International License. To view the details of this license, please visit (https://creativecommons.org/licenses/ by-nc-nd/4.0/).

(C) The Japanese Society of Internal Medicine Intern Med Advance Publication 